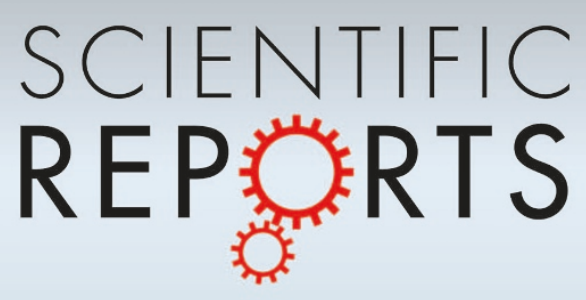

OPEN

SUBJECT AREAS:

ELECTRONIC DEVICES

APPLIED PHYSICS

ELECTRONIC AND SPINTRONIC

DEVICES

ELECTRICAL AND ELECTRONIC ENGINEERING

Received

6 August 2014

Accepted

7 October 2014

Published

28 October 2014

Correspondence and requests for materials should be addressed to A.V. (aapo.varpula@ vtt.fi) or M.P. (mika. prunnila@vtt.fi)

\title{
Harvesting Vibrational Energy Using Material Work Functions
}

\author{
Aapo Varpula, Sampo J. Laakso, Tahvo Havia, Jukka Kyynäräinen \& Mika Prunnila
}

VTT Technical Research Centre of Finland, P. O. Box 1000, 02044 VTT, Espoo, Finland.

Vibration energy harvesters scavenge energy from mechanical vibrations to energise low power electronic devices. In this work, we report on vibration energy harvesting scheme based on the charging phenomenon occurring naturally between two bodies with different work functions. Such work function energy harvester (WFEH) is similar to electrostatic energy harvester with the fundamental distinction that neither external power supplies nor electrets are needed. A theoretical model and description of different operation modes of WFEHs are presented. The WFEH concept is tested with macroscopic experiments, which agree well with the model. The feasibility of miniaturizing WFEHs is shown by simulating a realistic MEMS device. The WFEH can be operated as a charge pump that pushes charge and energy into an energy storage element. We show that such an operation mode is highly desirable for applications and that it can be realised with either a charge shuttle or with switches. The WFEH is shown to give equal or better output power in comparison to traditional electrostatic harvesters. Our findings indicate that WFEH has great potential in energy harvesting applications.

T

here is an on-going quest of efficient miniaturised energy harvesting devices that can harvest energy from their environment and supply sufficient power for autonomous operation of small electronic devices ${ }^{1}$. Energy harvesting is an especially attractive powering solution in applications where battery replacement or recharging is difficult or where continuous operation without maintenance is otherwise desirable. Such applications include, e.g., wireless sensor networks ${ }^{2-6}$ and biomedical implants $\mathrm{s}^{7-9}$. Energy harvesting schemes include, e.g., harvesting from ambient light, temperature gradients, and mechanical vibrations ${ }^{10}$. Due to abundant availability of mechanical vibrations in many relevant environments, vibration energy harvesters ${ }^{6,10-13}$ have gained large popularity. They convert mechanical vibrations into electricity using, e.g, electromagnetic induction, piezoelectricity, and electrostatics $s^{6,10,11}$. Furthermore, magnetostrictive materials ${ }^{11,14}$, magnetic shape memory alloys ${ }^{15}$, and triboelectrification ${ }^{16}$ have also been proposed for vibration energy harvesting.

This work is linked to electrostatic energy harvesters that are based on electrically charged capacitors, the capacitance of which is varied by mechanical motion. The mechanical energy is converted into electric energy of the capacitor, i.e., external force is performing work to increase the distance between charge on individual plates and thereby increasing the total potential energy of the charges. The fabrication of the electrostatic energy harvesters is straightforward as their structures are similar to other existing micro-electro-mechanical systems (MEMS). The drawback of the electrostatic energy harvesters is the need of electret materials, which pose challenges in the fabrication and lifetime, or an external power supply $y^{6,10,11,13,17}$. Due to these challenges piezoelectric energy harvesters, that can be easily fabricated also in microscale, have gained vast popularity in energyharvester research activities over the electrostatic harvesters. However, in addition to electrets and external batteries, there exists an alternative solution to introduce the initial charge to electrostatic harvester. This solution is given by a fundamental property of all materials: the work function. When two different materials with different work functions are brought in proximity to each other and connected galvanically, charging occurs naturally without any external source (Fig. la and b). This charging effect is based on the desire to reach global thermodynamic equilibrium, where the chemical potentials of the materials are equal. We refer the energy harvester utilizing the material work functions as the work function energy harvester (WFEH).

The charging effect between two bodies with different work functions was thoroughly characterized by Lord Kelvin already in $1861^{18,19}$. It plays an important and well-known role in many classical semiconductor devices ${ }^{20}$ and different solar-energy harvester $\mathrm{s}^{20-23}$. Despite this extensive history the use of work function difference in vibration energy harvesting has been, remarkably, proposed only quite recently ${ }^{24}$ and no experimental data exists $^{24-26}$. In this work, we present general model for WFEHs and experimental results, which validate the operating principle of the WFEH. Optimization of the WFEH device based on the presented ideal operating modes is provided and feasibility of the WFEH as a MEMS device is shown. Furthermore, the use of either a 

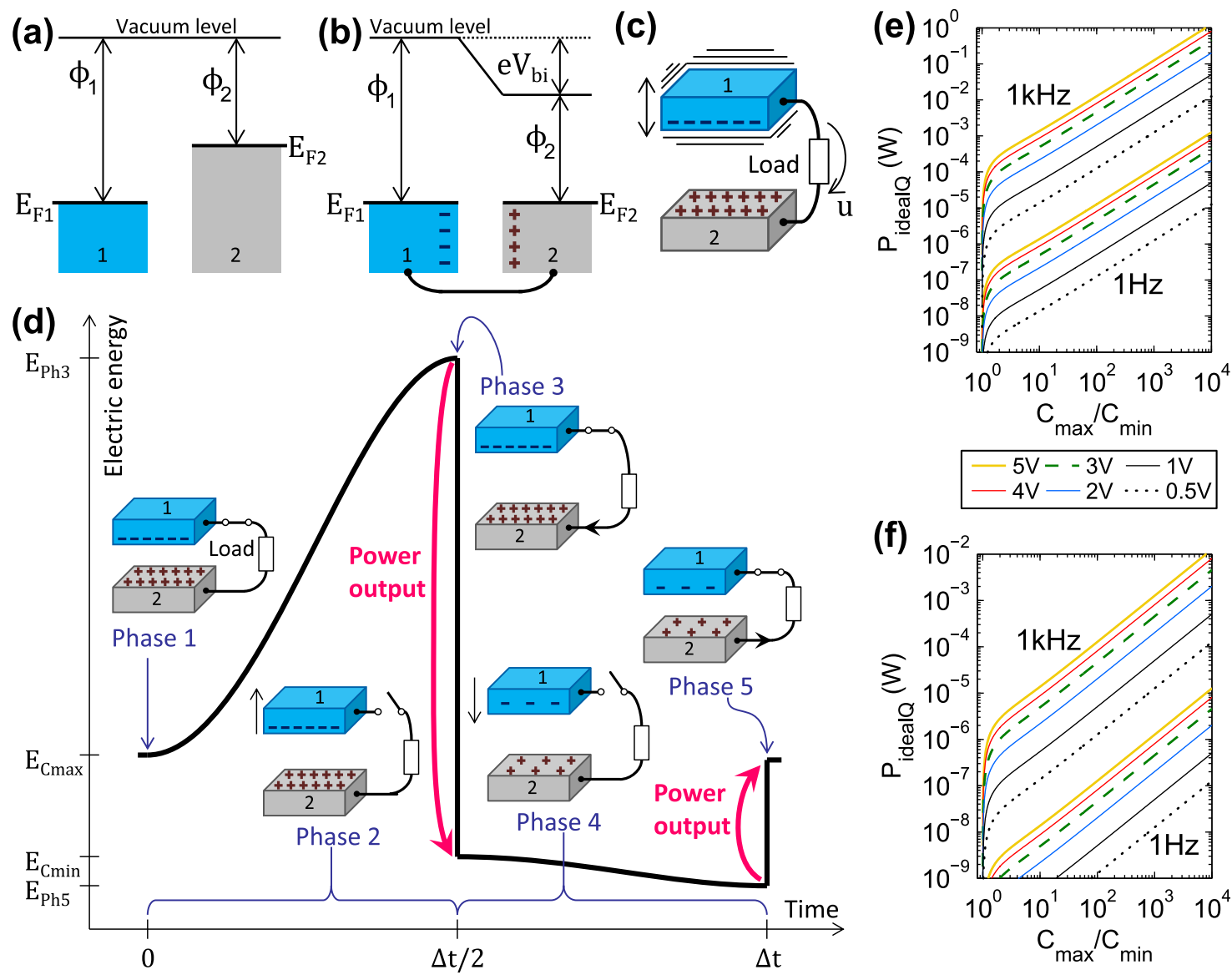

Figure $1 \mid(a, b)$ Electron energy levels of electrode materials 1 and 2 of the work-function energy harvester (a) before making contact and (b) in the thermodynamical equilibrium after making contact. $\mathrm{E}_{\mathrm{F} 1}$ and $\mathrm{E}_{\mathrm{F} 2}$ are the Fermi levels and $\phi_{1}$ and $\phi_{2}$ the work-functions of the materials 1 and 2 , respectively, $\mathrm{V}_{\mathrm{bi}}$ is the built-in voltage, and e is the elementary charge. Here the electrode materials are assumed to be metals, but semiconductors can be utilized as well. (c) Example of a WFEH generating output voltage u. (d) Phases of ideal cycle of a WFEH operating in the charge constrained mode: (1) initiation from the equilibrium conditions at $\mathrm{C}=\mathrm{C}_{\max },(2)$ decreasing $\mathrm{C}$ with the switch open, (3) discharging of the excess electric charge at $\mathrm{C}=\mathrm{C}_{\min }$, $(4)$ increasing $\mathrm{C}$ with the switch open, and (5) charging of the WFEH at $\mathrm{C}=\mathrm{C}_{\max }$. The graph shows schematically the variation of the electric energy stored in the WFEH during the cycle. Although the images depict a WFEH with varying distance of the gap, the ideal cycle applies to all WFEHs. $\mathrm{E}_{\mathrm{Cmin}}$ and $\mathrm{E}_{\mathrm{Cmax}}$ are the electric energies stored in the WFEH when the capacitance of the WFEH is at the minimum and maximum, respectively. $\mathrm{E}_{\mathrm{Ph} 3}$ and $\mathrm{E}_{\mathrm{Ph} 5}$ are the stored electric energies in the beginning of the phases 3 and 5, respectively. (e, f) Average powers of ideal work-function energy harvesters operated in the charge constrained mode with $\mathrm{C}_{\max }$ of (e) $10 \mathrm{nF}$ and (f) $100 \mathrm{pF}$ as functions of ratio $\mathrm{C}_{\max } / \mathrm{C}_{\min }$ and the built-in voltage $\mathrm{V}_{\mathrm{bi}}$. Data was calculated using equation (4) with $\mathrm{C}_{\mathrm{par}}=0$. The family of high power curves were calculated at the frequency of $1 \mathrm{kHz}$ and the family of low power curves at $1 \mathrm{~Hz}$.

charge shuttle or external switches to drive local electric energy storage for pulsed power supply is also described. We also show that the WFEH has equal or higher power output than a comparable electrostatic energy harvester. The similarity to the standard electrostatic energy harvesters allows similar device geometries and fabrication processes to be used and WFEHs can be fabricated with CMOS (complementary metal-oxide semiconductor) compatible materials. In fact, $\mathrm{n}$ and $\mathrm{p}$-type silicon (or other semiconductors) can be used as the different bodies of WFEH, indicating that the pn combo can also be used in vibration energy harvesters in addition to solar energy harvesters.

\section{Results}

Theory and ideal operating cycle. When two materials with different work functions are electrically connected, the Fermi levels are equalized by transport of electrons from the material with lower work function to the material with higher work function (see Fig. 1a and $b$ ). This displacement of electrons produces an excess of electrons and depletion of electrons, i.e. negative and positive electric charges, on the surfaces of the materials. These electric charges, in turn, give rise to an electric field and a voltage across the gap between the materials.

In the general case the voltage across the gap between the electrodes of the work-function energy harvester can be written as $V_{g a p}=u$ $V_{b i}$, where $\mathrm{u}$ is the output voltage of the $\mathrm{WFEH}$, i.e. voltage between the WFEH electrodes, measured via the connecting circuit (see Fig. 1c), $V_{b i}=\left(\phi_{1}-\phi_{2}\right) / e$ is the built-in voltage (i.e. the voltage across the gap in the thermodynamical equilibrium shown in Fig. 1b), e is the elementary charge, and $\phi_{1}$ and $\phi_{2}$ are the work functions of the electrode materials. The electric charge on the WFEH plates is given by $Q=C_{g a p} V_{g a p}+C_{p a r} u$, where $C_{\text {gap }}$ is the capacitance between the electrodes and $\mathrm{C}_{\mathrm{par}}$ is the parasitic capacitance. The total capacitance of the WFEH can be written as $C=C_{g a p}+C_{p a r}$. Since $C_{p a r}$ is constant, the electric current supplied or drawn by the WFEH can be written using the equations for $V_{g a p}$ and $Q$ as

$$
i=\frac{d Q}{d t}=\frac{d}{d t}(C u)-V_{b i} \frac{d C}{d t}
$$

Equation (1) is the fundamental equation describing the charging and discharging phenomena caused by the built-in voltage. The drive 
towards thermodynamical equilibrium (where Fermi levels are equal as shown in Fig. 1b) gives rise to the term $V_{b i} \frac{d C}{d t}$ which can be exploited in energy harvesting. The overall behaviour of the harvester device depends on the time evolution of $\mathrm{C}$ and the external circuit connected to the electrodes. The time evolution of the capacitance depends on the geometry and mechanics of the system and the electrostatic force (equation (8) in Methods section). Since equation (1) depends also on the time derivative of the product of $\mathrm{C}$ and $\mathrm{u}$, highly nonlinear behaviour is expected. The simplest form of electric load is a resistor. The electric current passing through the load resistor $\mathrm{R}$ is given by $\mathrm{i}=-\mathrm{u} / \mathrm{R}$ and equation (1) reduces to

$$
C \frac{d u}{d t}+u\left(\frac{d C}{d t}+\frac{1}{R}\right)=V_{b i} \frac{d C}{d t} .
$$

By considering the electric energy stored in the WFEH and neglecting all losses, the power output of an ideal WFEH can be calculated (the details are given in Section 1 of Supplementary Information). The output power depends strongly on how the electric charge is extracted from the WFEH. We consider two operation schemes: voltage and charge constrained modes. The charge-constrained mode is shown to generate much higher output power than the voltage-constrained mode. Therefore, the output power of the charge-constrained mode represents the theoretical maximum output power of the WFEH. The motion of the work-function energy harvester is assumed to be periodic with a period of $\Delta t$. During the cycle of operation the total capacitance of the WFEH is varied from the minimum value $\mathrm{C}_{\min }$ to the maximum value $\mathrm{C}_{\max }$.

In the voltage constrained mode any excess electric charge is immediately extracted using, for example, a low impedance load circuit. The voltage constrained mode yields the average output power as

$$
P_{\text {ideal } V}=\frac{C_{m a x} V_{b i}^{2}}{\Delta t}\left(1-\frac{C_{\min }}{C_{\max }}\right) .
$$

In the charge constrained mode the WFEH capacitor is charged or discharged only when the stored electric energy reaches a maximum or minimum, respectively. These extremes are reached when the WFEH capacitance is at maximum and minimum. The charging and discharging can be controlled with an electrical switch, for example. The ideal operating cycle of a WFEH operating in the charge constrained mode consist of 5 phases depicted in Fig. $1 \mathrm{~d}$. By combining all the phases of the cycle the average output power of a WFEH operating in the charge constrained mode can be written as

$$
\begin{aligned}
& P_{\text {ideal }}=\frac{C_{\text {max }} V_{b i}^{2}}{2 \Delta t}\left(1-\frac{C_{\text {min }}}{C_{\text {max }}}\right) . \\
& \left\{\frac{C_{\text {max }}}{C_{\text {min }}}+\frac{C_{\text {min }}}{C_{\text {max }}}+2-\frac{2 C_{\text {par }}}{C_{\text {min }}}\left[1+\left(\frac{C_{\text {min }}}{C_{\text {max }}}\right)^{2}\right]\right\} .
\end{aligned}
$$

Unlike in the charge-constrained mode, the ideal voltage-constrained mode does not lose power because of the parasitic capacitor. The ideal voltage-constrained mode could also be realized more easily, but comparison of equations (3) and (4) shows that the power output of the charge-constrained mode is at least two times the power output of the voltage-constrained mode. At large values of $\mathrm{C}_{\max } / \mathrm{C}_{\min }$ ratio the charge-constrained mode generates substantially more power than the voltage-constrained mode with limiting value $P_{\text {idealQ }}=C_{\text {max }} V_{b i}^{2}\left(C_{\text {max }} / C_{\text {min }}\right) / 2 \Delta t$.

The average powers of ideal work-function energy harvesters operated in the charge constrained mode are plotted in Fig. 1e-f as functions of $\mathrm{C}_{\max } / \mathrm{C}_{\min }$ ratio, frequency $\mathrm{f}=1 / \Delta \mathrm{t}$, and the built-in voltage $\mathrm{V}_{\mathrm{bi}}$. High output power can only be reached if $\mathrm{C}_{\max }$ is much larger than $C_{\min }$. In this case the power generated in phase 5 is negligible compared to the phase 3 (see Fig. 1d). As Fig. 1e-f and equation (4) show, $\mathrm{P}_{\text {idealQ }}$ is directly proportional to the operating frequency and the square of $\mathrm{V}_{\mathrm{bi}}$. Therefore, material pairs with high built-in voltages are desired, but otherwise any materials with sufficient conductivity are suitable and in addition to metals also semiconductors can be utilised. For example, for metal pair Pt and $\mathrm{Mg}$ we have $\mathrm{V}_{\mathrm{bi}}$ between $1.46 \mathrm{~V}$ and $2.27 \mathrm{~V}$, and for Pt and $\mathrm{Al}$ between $0.86 \mathrm{~V}$ and $1.87 \mathrm{~V}^{27}$. Here the spread in $\mathrm{V}_{\mathrm{bi}}$ arises from the typical spread in the work function values of metals found in the literature. If the material pair is formed from the same semiconductor but of different type ( $\mathrm{p}$ and $\mathrm{n}$ ), then the built-in voltage is roughly equal to the energy gap of the semiconductor. For example, a built-in voltage of $1.1 \mathrm{~V}$ could be obtained with silicon and values above $3 \mathrm{~V}$ could be reached with wide band-gap semiconductors such as $\mathrm{SiC}$ and $\mathrm{GaN}$. Availability of $\mathrm{n}$ and $\mathrm{p}$-type diamond allows built-involtages of over $5 \mathrm{~V}$ to be reached.

\section{Experimental verification with prototype of work-function energy} harvester. In order to verify the model and to demonstrate the work function energy-harvesting concept we built a macroscopic variable capacitance prototype with $63 \mathrm{~mm} \times 64 \mathrm{~mm}$ aluminium and copper capacitor plates (see Fig. $2 \mathrm{a}$ and $\mathrm{b}$ and Methods). In the harvesting test measurements a voltage across a load resistor was recorded against temporal change of the $\mathrm{Al}-\mathrm{Cu}$ capacitor. Prior to these measurements the built-in voltage of the plates was measured using a null method, where the load and the measurement instrument is replaced by a DC voltage source $U_{D C}$ and the output current is measured while the capacitor plates move. In this configuration, similar to the one invented by Lord Kelvin ${ }^{18,19}$, equation (1) reduces to $i=\left(U_{D C}-V_{b i}\right) d C / d t$. The RMS of measured current $i$ as a function of $U_{D C}$ is shown in Fig. $2 \mathrm{~d}$ and the RMS current is minimized at $U_{D C}=V_{b i}$, which is given by the intersection of the lines fitted to the data. The average value of the measured built-in voltage $\mathrm{V}_{\mathrm{bi}}$ was $1.03 \pm 0.08 \mathrm{~V}$.

The time dependencies of the measured and simulated voltage across a load resistor connected to the experimental work-function energy harvester with different minimum distances between the plates $\mathrm{d}_{0}$ (see equations (5) and (6) in Methods) and different load resistors are shown in Fig. 2e and $f$ and Supplementary Figs. 1 and 2. In order to compare the data obtained at various operating frequencies $1 / \Delta t$ in a single plot, the normalized time $t / \Delta t$ is used. The total capacitance is at maximum at time $t=\Delta t / 2$ and at minimum at times $\mathrm{t}=0$ and $\mathrm{t}=\Delta \mathrm{t}$. The data shows that the experiments and the numerical model agree well over the whole range of frequencies $(10 \mathrm{mHz}-0.9 \mathrm{~Hz})$ and values of $\mathrm{d}_{0}(170 \mu \mathrm{m}, 350 \mu \mathrm{m}$, and $580 \mu \mathrm{m})$ and different loads (1 G $\Omega$ and $100 \mathrm{M} \Omega$ ). The shape of the voltage signal changes from signal having two peaks with opposite polarities to smoothed triangle wave as the frequency increases and $\mathrm{d}_{0}$ decreases. At the same time the average output power of the WFEH increases. These effects are caused by the fact that the operating period $\Delta \mathrm{t}$ approaches the electrical time constant $\mathrm{RC}_{\max }$ of the WFEH.

The feasibility of using the simple load resistor as the harvesting circuit can be estimated by comparing the average output power of the system $\mathrm{P}_{\text {ave }}$ to the output power of an ideal WFEH operating in the charge-constrained mode, $\mathrm{P}_{\text {idealQ }}$ (equation (4)), as this mode generates the highest obtainable power. The measured and simulated normalized powers $\mathrm{P}_{\text {ave }} / \mathrm{P}_{\text {idealQ }}$ are plotted in Fig. 3a as functions of the operating frequency. At low frequencies and large values of $\mathrm{d}_{0}$ the measured output powers are slightly larger than the simulated powers due to noise. The highest measured power was $10 \%$ of the ideal power. In Section 3.2 of Supplementary information it is shown that a WFEH with load resistor is characterized by the normalized time constant $\mathrm{RC}_{\max } / \Delta \mathrm{t}$. Normalizing the operating frequencies of the measured data (see Fig. $3 \mathrm{~b}$ ) shows, that the features of the power curves indeed fall on almost the same locations on the $\mathrm{RC}_{\max } / \Delta \mathrm{t}$ axis. 

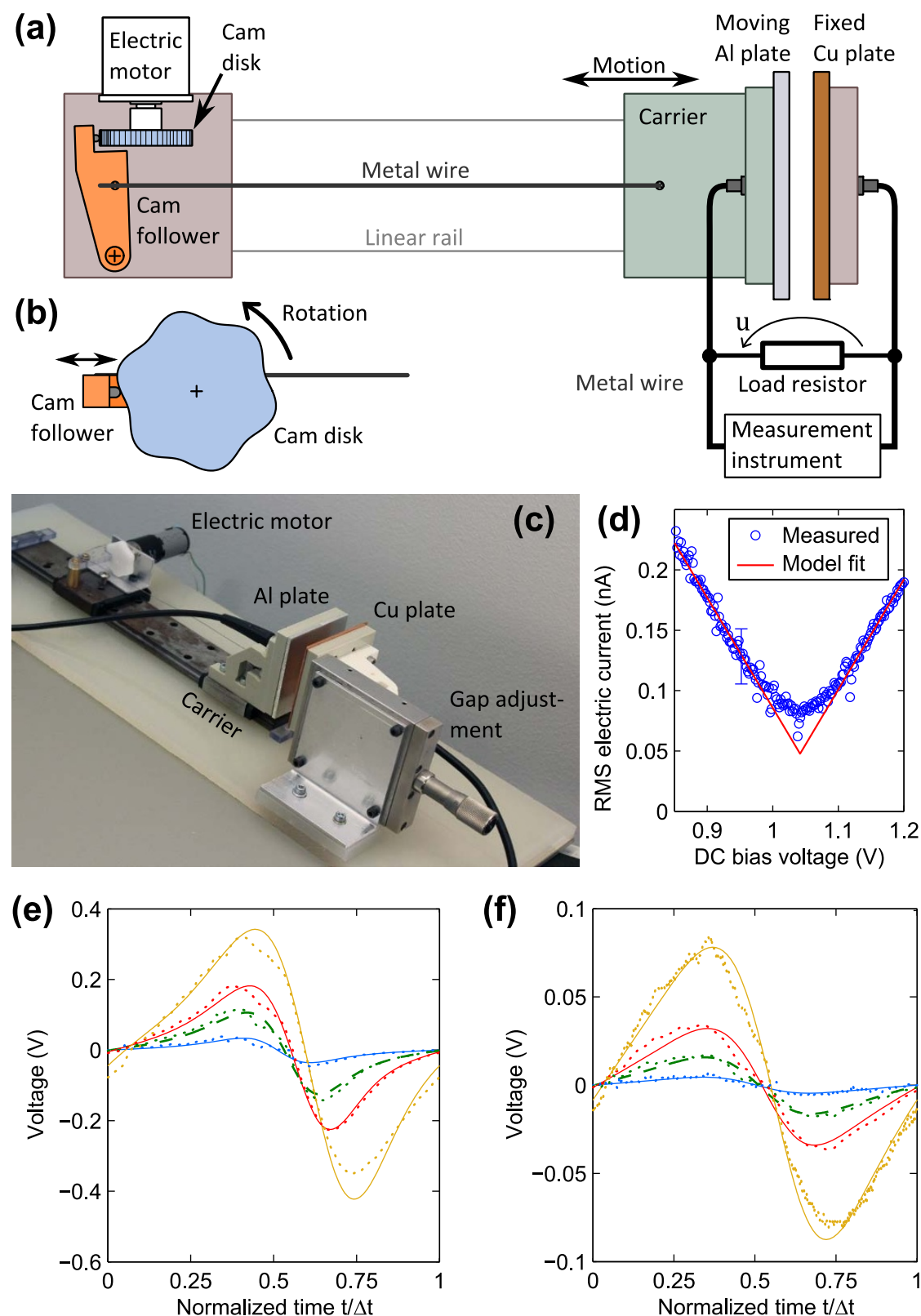

\begin{tabular}{|lllll|}
\hline & $0.05 \mathrm{~Hz}$ & $0.2 \mathrm{~Hz}$ & $0.3 \mathrm{~Hz}$ & $0.9 \mathrm{~Hz}$ \\
Measured & $\cdots \cdots \cdots$ & $\cdots \cdots \cdots$ & $\cdots \cdots \cdots$ & $\cdots \cdots$ \\
Simulated & & $-\ldots-$ & & \\
\cline { 3 - 4 } & & &
\end{tabular}

Figure $2 \mid$ (a,b) Schematic picture of the experimental setup: (a) top view of the whole setup and (b) side view of the cam disk. The setup is inclined at an angle of 15 degrees in order to utilize gravitational counter force. Voltage $u$ across the load resistor is indicated. (c) Photograph of the setup. The initial gap between the plates is adjusted with a positioner stage. (d) Example of electric current data from DC voltage sweeping measurements. The value of the built-in voltage $V_{\mathrm{bi}}=1.03 \pm 0.08 \mathrm{~V}$ is determined from the intersection of the lines fitted to the data. The RMS value of the electric current is not equal to zero at $\mathrm{u}=\mathrm{V}_{\mathrm{bi}}$ due to noise and current offset. (e, f) Time dependencies of measured and simulated voltage across a $1.00 \mathrm{G} \Omega$ load resistor connected to a $\mathrm{Cu} / \mathrm{Al}$ parallel plate work-function energy harvester with the minimum distance between the plates $\mathrm{d}_{0}$ of (e) $170 \mu \mathrm{m}$ and (f) $580 \mu \mathrm{m}$. Time is normalized with the operating period $\Delta \mathrm{t}$.

The differences between the curves are caused by different values of $\mathrm{C}_{\max } / \mathrm{C}_{\min }$, which range from 1.9 to 4.0 .

Optimisation of output power using variable load. The temporal variation of capacitance has a large effect on the performance of a WFEH (see Section 3.3 of Supplementary information). For a specific temporal dependence of the capacitance there are optimal values for $\mathrm{RC}_{\max } / \Delta \mathrm{t}$ and $\mathrm{C}_{\max } / \mathrm{C}_{\min }$. A WFEH with a resistor as the load generates less output power than both the charge-constrained and voltage-constrained operating cycles. This is caused by non-optimal charging and discharging of the WFEH capacitor. For maximum power the WFEH capacitor should be fully charged when the capacitance is at the maximum and fully discharged when the capacitance is at the minimum. At low frequencies the WFEH capacitor is discharged too early because of the slow variation of the WFEH capacitance. At high frequencies, in turn, the WFEH operates too quickly to be adequately charged when the WFEH capacitance is at its maximum. Fig. 3 illustrates well this behaviour. 

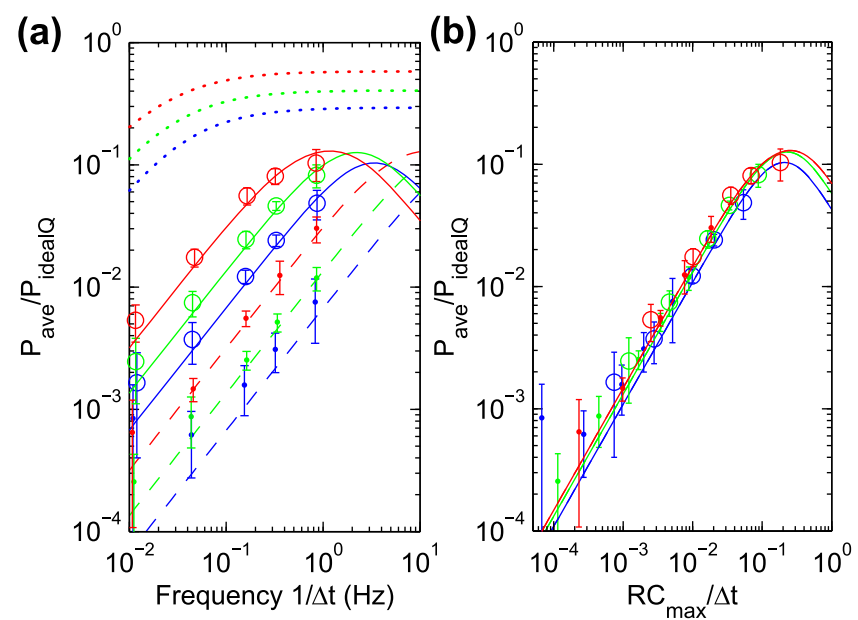

\begin{tabular}{|c|c|c|c|}
\hline $170 \mu \mathrm{m}, 1 \mathrm{G} \Omega$ & Measured & Simulated & $\begin{array}{l}\text { Simulated, } \\
\text { variable load }\end{array}$ \\
\hline $170 \mu \mathrm{m}, 100 \mathrm{M} \Omega$ & • & --- & $\cdots \cdots \cdots 170 \mu \mathrm{m}$ \\
\hline $350 \mu \mathrm{m}, 1 \mathrm{G} \Omega$ & 0 & $\longrightarrow$ & $\cdots \cdots 350 \mu \mathrm{m}$ \\
\hline $350 \mu \mathrm{m}, 100 \mathrm{M} \Omega$ & - & --- & $\cdots \cdots \cdots 580 \mu \mathrm{m}$ \\
\hline $580 \mu \mathrm{m}, 1 \mathrm{G} \Omega$ & 0 & $\longrightarrow$ & \\
\hline $580 \mu \mathrm{m}, 100 \mathrm{M} \Omega$ & • & --- & \\
\hline
\end{tabular}

Figure 3 Measured and simulated average output power $\mathrm{P}_{\text {ave }}$ of a $\mathrm{Cu} / \mathrm{Al}$ parallel plate work-function energy harvester with a load resistor $R$ as a function of (a) the operating frequency $1 / \Delta t$ and (b) the normalized time constant $\mathrm{RC}_{\text {max }} / \Delta \mathrm{t}$. The average output powers are normalized to the ideal charge-constrained output power $\mathrm{P}_{\text {idealQ }}$. The value of the load resistor and the minimum distance between the plates $\mathrm{d}_{0}$ are indicated in the legend. Simulated data of a WFEH with a load varying between on-state $(10 \Omega)$ and off-state (100 G $\Omega)$ is also shown in (a).

The determining factor is the ratio of the electrical time constant, $\mathrm{RC}_{\text {max }}$, and the period of the mechanical motion, $\Delta \mathrm{t}$. Between these two extremes, when ratio $\mathrm{RC}_{\max } / \Delta \mathrm{t}$ is near 0.2 , the optimal operation is reached.

The ideal charge-constrained cycle defines the maximum output power of the work-function energy harvesters. This ideal cycle requires use of variable load in the harvesting circuit. Variable load can be realized using, for example, electrical switches. Such switching circuits ${ }^{28-30}$ have already been utilized in electrostatic energy harvesters, the operation of which is similar to the WFEHs. The switching ensures that the WFEH capacitor is fully charged and discharged during the operation cycle similarly as in the ideal cycle. In the simplest case the WFEH can be charged by shorting the capacitor electrodes. This can be done using a mechanical switch in parallel with the load resistor. The timing of the switching is critical as the switch should only be closed when the capacitance is at its maximum, otherwise the output power can even be much lower than the power obtained without switching. Despite this difficulty, we managed to demonstrate over 2-fold increase in power output of our experimental setup using the switching operation (see the details in Section 2.2 of Supplementary information).

The power increase due to the use of a variable load in workfunction energy harvesters estimated with our numerical model is shown in Fig. 3a. The normalized power decreases with decreasing frequency because the off-state resistance $(100 \mathrm{G} \Omega)$ is not high enough to avoid leakage current. The simulations with variable loads yield much higher power than the simulations with fixed load: With the minimum plate distance of $170 \mu \mathrm{m}$ the simulated variable load WFEH produces $58 \%$ of the ideal power at maximum, whereas the simulated fixed load WFEH produces only $13 \%$ of the ideal power at maximum.
Estimation of performance of MEMS device. The performance of a MEMS-based WFEH device can be estimated by using MEMS-scale parameter values of electrostatic energy harvesters. Here we adopt the parameters of Ref. 31. In the MEMS simulations (see Methods) the energy harvester was connected to either constant or variable load and excited with sinusoidal vibration with an amplitude of $a$ $=32.5 \mathrm{~m} / \mathrm{s}^{2}$ around the mechanical resonance frequency $f_{\mathrm{r}}=$ $1868 \mathrm{~Hz}$. With this kind of excitation and a charging voltage of $9 \mathrm{~V}$ the electrostatic harvester gives an output power of $1.2 \mu \mathrm{W}^{31}$. The dependence of the output power of the simulated WFEH device on the frequency of the vibration is shown in Fig. 4a. Note that by setting the built-in voltage of the WFEH and the external power supply of electrostatic harvester equal we find that the WFEH gives equal or higher power output (see Section 5 of Supplementary information). The output power of the WFEH increases with increasing value of the built-in voltage $\mathrm{V}_{\mathrm{bi}}$. The device produces the highest power when the vibration frequency matches with the mechanical resonance frequency of the device. Comparison of the data from the devices with constant and variable loads shows that the output power increases when switching is used. For example, at the built-in voltage of $1 \mathrm{~V}$ the increase in output power is over one order of magnitude. The power maxima vs. frequency in the variable load case are wider than in the fixed load case. In other words, the harvesting efficiency in the variable load case is less sensitive to matching the mechanical resonance frequency to the vibration frequency, which underlines the importance of electrostatic force (equation 8 in Methods section) in the dynamics.

The dependence of the output power on the built-in voltage is shown in Fig. 4b. In the case of constant $200 \mathrm{k} \Omega$ load, the power increases quadratically with $\mathrm{V}_{\mathrm{bi}}$, as suggested by equations (3) and
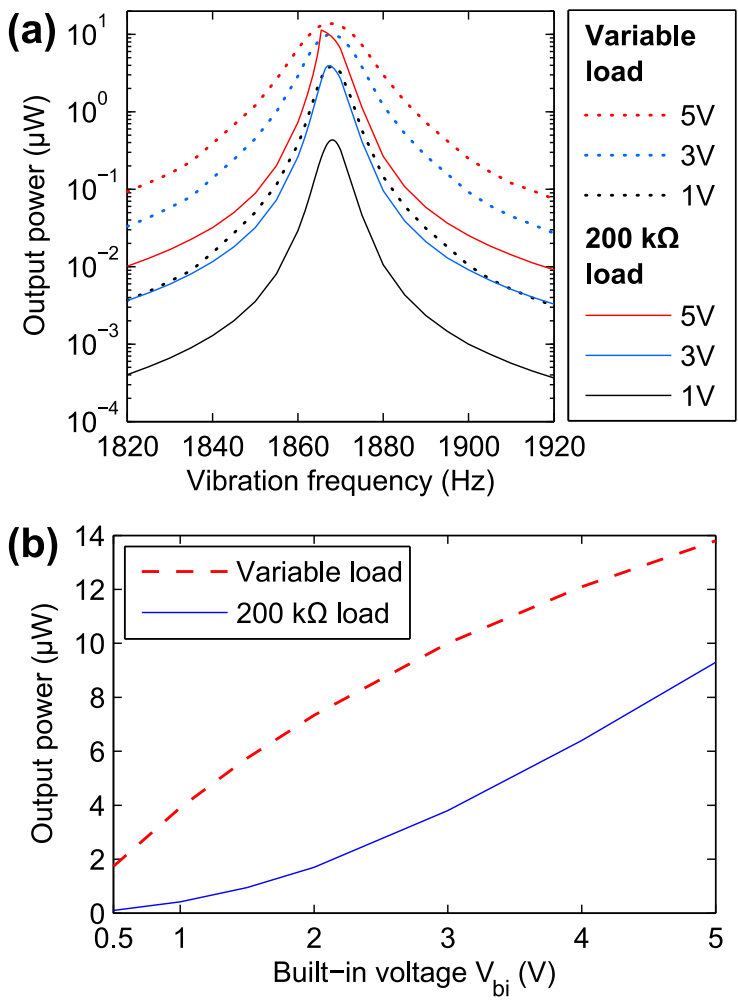

Figure 4 | Simulated output power of MEMS work-function energy harvesters with a load varying between $100 \Omega$ and $50 \mathrm{G} \Omega$ and a constant $200 \mathrm{k} \Omega$ load as (a) functions of the vibration frequency at three values of $\mathrm{V}_{\mathrm{bi}}$ and (b) function of the built-in voltage $\mathrm{V}_{\mathrm{bi}}$ at vibration frequency of $1868 \mathrm{~Hz}$. The parameters of the simulated device were taken from an existing electrostatic energy harvester ${ }^{31}$. The amplitude of the vibration is $32.5 \mathrm{~m} / \mathrm{s}^{2}$ 
(4), but in the case of variable load the dependence is weaker (the second derivative of the curve is negative). This is likely a consequence of electrostatic spring softening, which shifts the resonance off the exciting vibration frequency. In the constant load case this effect is not observed because the duration of the peak electrostatic force per cycle is shorter than in the variable load case.

Work-function charge pump. In the most effective part of the charge-constrained ideal cycle (Fig. 1d) the WFEH acts essentially as a charge pump. It pushes the excess charge to an external circuit. This can be exploited in an elegant manner to charge a storage capacitor $\mathrm{C}_{\text {sto }}$, which, in turn, can supply power to an electric load. Such a work-function charge pump can be constructed from a shuttle moving between the fixed electrodes as sketched in Fig. 5a. During the operation of this device (see Fig. 5b) the shuttle first makes electric contact with the lower electrode, thus charging the shuttle. Next, the shuttle moves up and the electric energy in the system increases as the capacitance between the shuttle and the lower electrode decreases. Finally, the shuttle touches the upper electrode and the excess electric charge in the shuttle flows to $\mathrm{C}_{\text {sto. }}$. Note that the work-function charge shuttle has a captivating analogy with a nanomechanical single-electron shuttle ${ }^{32}$. The shuttle operation can be adapted for WFEH in general or, on the other hand, a workfunction charge pump can be realized without a shuttle using external switches (see Fig. 5c) that mimic the phases of Fig. 1d. In principle, the switch configuration should exhibit characteristics identical to that of the shuttle device, but the shuttle device can provide significantly larger maximum capacitance.

Despite the differences in their operating principles and designs, the characteristics of the work-function charge pumps of Fig. 5a and c can be described by the same model, where the stored charge after $\mathrm{N}$ pumping cycles is derived using the formula for a geometric sum (see detailed derivation in Section 4 of Supplementary information). The time dependence of the electric energy $E_{\text {sto }}=C_{\text {sto }} u_{\text {sat }}^{2}\left\{1-\left[C_{\text {sto }} /\left(C_{\text {sto }}+C_{\text {min }}\right)\right]^{N}\right\}^{2} / 2$ accumulated in the storage capacitor $\mathrm{C}_{\text {sto }}$ is shown in Fig. $5 \mathrm{~d}$, where the normalized electric energy is plotted as a function of the normalized number of charging cycles $\mathrm{N} / \mathrm{N}_{\mathrm{mpp}}$. Here $\mathrm{u}_{\mathrm{sat}}=-\left(\mathrm{C}_{\max } / \mathrm{C}_{\min }-1\right) \mathrm{V}_{\mathrm{bi}}$ is the saturation voltage (i.e. maximum output voltage of the WFEH). After charging $\mathrm{N}_{\mathrm{mpp}}=\ln (2) / \ln \left(1+\mathrm{C}_{\text {min }} / \mathrm{C}_{\text {sto }}\right)$ cycles the charging power $P_{\text {sto }}=C_{\text {sto }} \ln \left[C_{\text {sto }} /\left(C_{\text {sto }}+C_{\text {min }}\right)\right]\left(u_{\text {sto }}-u_{\text {sat }}\right) u_{\text {sto }} / \Delta t$ reaches a maximum of $P_{\text {sto }}^{\max }=C_{\text {sto }} u_{\text {sat }}^{2} \ln \left(1+C_{\text {min }} / C_{\text {sto }}\right) / 4 \Delta t$. At the maximumpower point $25 \%$ of the maximum electric energy is stored in $C_{\text {sto. }}$. Fig. 5d shows that in the beginning the stored energy increases slowly with each cycle. When the energy in the storage capacitor approaches the maximum, the energy increase per cycle decreases rapidly. These effects are caused by the dependence of the charging power on the voltage $\mathrm{u}_{\text {sto }}$, which is shown in Fig. 5e. In the beginning most of the energy is lost due to the low value of $u_{\text {sto }}$. The charging power increases as $\mathrm{u}_{\text {sto }}$ increases. After $\mathrm{P}_{\text {sto }}$ reaches a maximum, it begins to decrease due to the fact that the stored charge corresponding to the high value of $\mathrm{u}_{\text {sto }}$ prevents flow of charge from the WFEH to the storage capacitor. For the same reason, the charging power decreases when the value of $\mathrm{C}_{\min }$ approaches the value of $\mathrm{C}_{\text {sto. }}$. In practical applications $\mathrm{C}_{\text {sto }}$ is much larger than $\mathrm{C}_{\min }$. In this range $P_{\text {sto }}^{\max }$ reduces to $P_{\text {sto }}^{\max } \approx C_{\text {max }} V_{b i}^{2}\left(C_{\text {max }} / C_{\text {min }}\right)\left(1-C_{\text {min }} / C_{\text {max }}\right)^{2} / 4 \Delta t$. Comparing this formula and equation (4) in the case that $C_{\max }$ is much larger than $\mathrm{C}_{\min }$ shows that the maximum charging power of the system is half of the corresponding ideal power of the charge constrained mode $\mathrm{P}_{\text {idealQ}}$. This is due to the fact that at the maximum power point the WFEH capacitor cannot be discharged by more than half of its capacity.

\section{Discussion}

Work-function energy harvesters are similar to the electrostatic energy harvesters as they both employ the same principle of energy conversion with variable capacitance. Therefore, the device geometries and various broadband vibration energy harvesting techniques utilized in electrostatic energy harvesters ${ }^{6,33,34}$ can also be used in WFEHs. The key advantage of the work function energy harvester over the electrostatic energy harvester is the fact that the work function energy harvester does not need external power source or electret materials in the operation. In this sense the WFEH becomes closer to piezoelectric harvesters that also rely on fundamental properties of solid materials. Another unique feature of WFEH is that it can be operated in the charge shuttle mode, which is impossible to realise with electrostatic harvesters. The charge shuttle mode can provide extremely large maximum capacitance and, therefore, high output power. The physical contact between the surfaces, however, often results in stiction problems and reduced lifetime, but recently reported $\mathrm{SiC}$-based nanoelectromechanical switches ${ }^{35}$ have been shown to operate reliably over $10^{7}$ switching cycles.

WFEHs can generate more power than the electrostatic harvesters in many operating conditions (see Section 5 of Supplementary information). A simple comparison can be performed by assuming that the initial charging voltage $\mathrm{V}_{\text {in }}$ of the electrostatic harvester is equal to the built-in voltage $V_{b i}$ of the WFEH. A more detailed comparison would need the details of the specific applications of the devices. In principle, high values of $V_{\text {in }}$ can be used in the electrostatic harvester, since the charging voltage is limited only by the pull-in voltage and the breakdown voltage of the variable capacitor of the device. In reality, the charging voltage is limited by the available power supply, which is a battery with the nominal voltage $1-4 \mathrm{~V}$ in many applications. This voltage range can also be realized with the built-in voltages of many known material pairs.

In general, energy harvesters can be operated in two modes: continuously and in pulsed mode. In the pulsed mode the WFEH needs to supply energy to, for example, sensing and communicating circuit which measures and transmits signals for further processing at time intervals. The use of a work-function charge pump of either Fig. 5a or $c$ in both of these operating modes can be designed using the results shown in Fig. 5d and e. The charging power can be maximized by selecting large storage capacitors, so that $C_{\text {sto }} / C_{\text {min }} \geq 100$. Workfunction charge-pump devices need an initialization time where they gather electric energy in the storage capacitor while increasing its charging power as the voltage across the storage capacitor increases. The number of operating cycles $\mathrm{N}_{\text {mpp }}$ needed to reach the maximum charging power is proportional to $\mathrm{C}_{\text {sto }} / \mathrm{C}_{\text {min }}$. In the pulsed mode the time interval between the pulses can be optimized so that the electric energy in the storage capacitor is not completely consumed, causing the charging power to collapse. On the other hand, unlike in the case of electrostatic harvesters using external battery all energy of the storage capacitor can be consumed during every cycle if needed. Finally, we note that a supercapacitor can provide an attractive solution to achieve high capacitance energy storage ${ }^{36}$ that can be integrated with WFEH.

In conclusion, we have experimentally tested the work-function energy harvester concept. The operation of the test device was in a good agreement with our theoretical model. Based on the theory two ideal modes of operation were devised. The charge-constrained mode was shown to produce much higher output power than the voltage-constrained mode. The results show that for maximum output power the electrical time constant of the work-function energy harvester should be optimized in respect to the operating period of the device. Use of switches or a charge shuttle in mimicking the ideal cycle was shown to increase the output power of the WFEH remarkably. We have also presented two work-function charge pump designs for charging of storage capacitors for powering of, e.g., autonomous sensors. The feasibility of miniaturizing work function energy harvesters was shown by simulating a realistic MEMS device. Finally, the comparison of work function and electrostatic energy harvesters showed that work function energy har- 

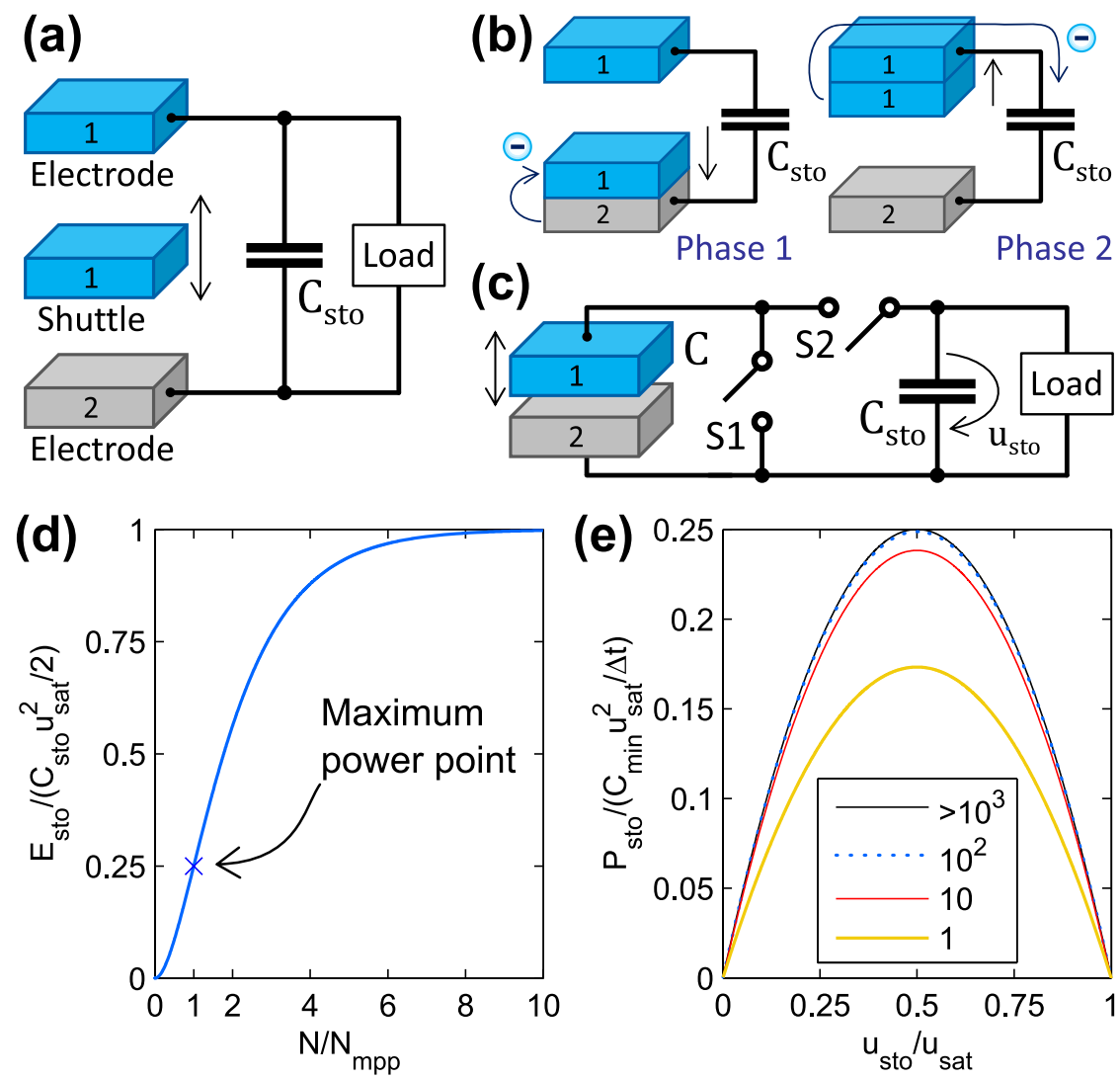

Figure $5 \mid$ (a) Schematic picture of a work-function charge pump, where a shuttle moving between two fixed electrodes acts simultaneously as a built-in switch. The shuttle can be fixed to a frame of reference, e.g., by an elastic beam (not shown). The shuttle and the upper electrode are made of the same material (1) and the lower electrode of material with dissimilar work function (2). This charge pump is connected to storage capacitor $\mathrm{C}_{\text {sto }}$, which supplies power to an electric load. (b) The operation phases of the work-function charge pump of (a): Charging of the shuttle (1) and the flow of charge to $\mathrm{C}_{\text {sto }}(2)$. (c) A work-function charge pump based on external switches S1 and S2: A work-function energy harvester with total capacitance C connected to storage capacitor $\mathrm{C}_{\text {sto }}$, which supplies power to a load circuit. $\mathrm{u}_{\text {sto }}$ is the voltage across $\mathrm{C}_{\text {sto. }}$. (d) Dependence of the normalized electric energy stored in $\mathrm{C}_{\text {sto }}$ as a function of the number of charging cycles $\mathrm{N}$ normalized with the number of cycles $\mathrm{N}_{\text {mpp }}$ needed to reach the maximum power point. The stored electric energy, $E_{s t o}$ is normalized with the maximum electric energy which can be stored in the storage capacitor, $E_{s t o}^{\max }=C_{\text {sto }} u_{\text {sat }}^{2} / 2$. (e) Dependence of the normalized charging power of a work-function charge pump on the normalized voltage $\mathrm{u}_{\text {sto }} / \mathrm{u}_{\text {sat }}$ calculated with various values of $\mathrm{C}_{\text {sto }} / \mathrm{C}_{\text {min }}$. The charging power is normalized with $C_{\min } u_{\text {sat }}^{2} / \Delta t$, which is twice the maximum average power available from the WFEH.

vesters can generate more power than the electrostatic harvesters in many operating conditions, but without the need of external power source or electret materials. This was also confirmed with the MEMS simulations. The results presented in this article point out that the work function energy harvesters have vast potential to be used in many applications. For maximum power output material pairs with maximal difference in their work functions need still to be sought and MEMS versions of the work function energy harvester need to be realized. The principle, however, does not require realization in microscale, but miniaturization is needed in many applications and achieving high capacitances is easier with microfabrication techniques. The optimal design of these MEMS devices can be achieved by utilizing the models presented in this article.

\section{Methods}

Experiments with WFEH prototype. The WFEH prototype is a parallel plate capacitor with the plates moving along an axis perpendicular to both plates (i.e. varying distance between the plates). The total capacitance of such a system is given by

$$
C(t)=\frac{\varepsilon_{0} A}{d(t)}+C_{p a r}
$$

where $\varepsilon_{0}$ is the vacuum permittivity (assuming air or vacuum between the plates), $d(t)$ the distance between the plates, and $A$ the area of the plates. The motion of the plates of the experimental setup is close to the ideal sinusoidal motion given by

$$
d(t)=\Delta \mathrm{d}\left[1-\cos \left(2 \pi \frac{t}{\Delta t}\right)\right]+d_{0},
$$

where $\Delta \mathrm{d}$ is the amplitude of the motion of the plates and $\mathrm{d}_{0}$ is the minimum distance between the plates.

The copper plate of the prototype is fixed and the aluminium plate is mounted on a carrier moving on a linear rail tilted by 15 degrees (see Fig. $2 \mathrm{a}$ and $\mathrm{b}$ ). The carrier is actuated with a DC motor and reduction gear that work against gravity. The rotation of the motor is converted to reciprocating linear motion using a cam disk made of polyoxymethylene (POM) and a cam follower with a steel tip. A metal wire connects the cam follower to the carrier. The sinusoidal motion of the plates was achieved using a cam disk whose radius varies sinusoidally.

Prior to experiments the $\mathrm{Al}$ and $\mathrm{Cu}$ plates were sanded, washed, and cleaned with isopropyl alcohol. In the measurements we utilized an Agilent $4156 \mathrm{C}$ precision semiconductor parameter analyzer operated using MathWorks ${ }^{\circledR}$ MATLAB ${ }^{\circledR}$ program via IEEE-488 interface. The plates were connected to the measurement instrument using spring and BNC connectors and flexible coaxial cables. The value of $0.25 \mathrm{~mm}$ was used for the amplitude of the motion $\Delta \mathrm{d}$ in all the measurements.

In the built-in voltage measurements the load resistor shown in Fig. 2a was removed and the plates were connected directly to $4156 \mathrm{C}$ instrument. The copper plate was connected to a source-measure unit (SMU) of $4156 \mathrm{C}$ instrument set at zero current and the aluminium plate was connected to a SMU set at a specified constant voltage $\mathrm{U}_{\mathrm{DC}}$. The electric current i supplied by the latter SMU was measured. The applied voltage $U_{D C}$ was swept and the root-mean-square (RMS) current was measured. The value of the built-in voltage is determined from the minimum of the RMS current as equation $i=\left(U_{D C}-V_{b i}\right) d C / d t$ shows. Integration time of $0.2 \mathrm{~ms}$ was used in the current measurement. $50 \mathrm{~Hz}$ and $150 \mathrm{~Hz}$ interference was filtered out of the measured current before calculating the RMS value. The plates were moved at the frequency of $3 \mathrm{~Hz}$ in order to increase the magnitude of the signal. 
The measurement circuit shown in Fig. 2a was used to measure the voltage across the load. The aluminium plate was connected to a SMU set to operate as voltage source at zero voltage. The copper plate was connected to a SMU operating as current source and was set at zero current. The voltage across the load resistor was obtained by reading the voltage measured by the SMU connected to the copper plate. When the frequency of motion of the plates was higher than $0.4 \mathrm{~Hz}$, the integration time of $0.2 \mathrm{~ms}$ was used. In order to remove the interference mainly from the power lines, $50 \mathrm{~Hz}$ and $150 \mathrm{~Hz}$ signals were digitally filtered out from the measured voltage. At low frequencies of motion the integration time of $20 \mathrm{~ms}$ (corresponding to the power line frequency) was used without digital filtering.

The capacitance dependence estimated by equations (5) and (6) was in agreement with measurements performed with a HP4192A impedance analyzer. However, an envelope function with a period of $6 \Delta \mathrm{t}$ was observed in the capacitance signal. More detailed modelling of the capacitance suggests that this envelope originates from the fact that the centre of the axis of the drive wheel is misaligned by $50 \mu \mathrm{m}$. In this kind of setup the elimination of the misalignment is practically impossible. On the other hand, adding more complexity to the capacitance model can cause problems in the extraction of the model parameters during model fitting. Therefore, in the measurements we used only data from cycles where the effect of the envelope function was low. The capacitance data obtained with the impedance analyser cannot directly be utilized in the harvesting test measurements because of differences in the measurement circuits. Because of this, the value of $0.1 \mathrm{pF}$ was obtained for the parasitic capacitance $\mathrm{C}_{\mathrm{par}}$ by model fitting.

Simulations. In general, vibration energy harvesters can be described as mechanical second-order spring-mass systems $s^{6,10,11,13}$, where the conversion of kinetic energy to electrical energy is taken into account as damping. The differential equation of motion of such a device is given by

$$
m \frac{d^{2} x}{d t^{2}}+b \frac{d x}{d t}+k x=m a+F_{e}
$$

where $\mathrm{m}$ is the proof mass, a is the acceleration of the proof mass, $\mathrm{k}$ the spring constant, and $\mathrm{b}$ the intrinsic damping coefficient. The electrostatic force given $b^{37}$

$$
\vec{F}_{e}=\frac{1}{2} V_{\text {gap }}^{2} \vec{\nabla} C_{\text {gap }},
$$

which acts on the capacitor $C_{\text {gap }}$ in the direction of the spatial gradient $\nabla C_{p a r}$. The time evolution of the capacitance depends on the geometry and mechanics of the system and the electrostatic force (equation (8)).

The WFEH prototype was modelled using capacitance dependence given by equations (5) and (6). The effect of the electrostatic force (equation (8)) was neglected. The numerical model of the WFEH connected to a circuit with resistor load (equation (2)) was solved using MathWorks ${ }^{\circledR}$ Simulink ${ }^{\circledR}$. The model was fitted to the experimental data using the MATLAB ${ }^{\circledR}$ function fminsearch. The minimum distance between the plates $d_{0}$ and the parasitic capacitance $C_{\text {par }}$ were used as the fitting parameters. Temporal offset and a small offset in voltage $(<1 \mathrm{mV})$ was allowed in the fitting. After initial set of experiments the value of $\mathrm{C}_{\mathrm{par}}$ was fixed to $0.1 \mathrm{pF}$.

In the numerical calculations with variable load the load was in the on-state $(10 \Omega)$ when $\mathrm{C}-\mathrm{C}_{\min }$ was higher than $99 \%$ and lower than $0.2 \%$ of the absolute capacitance change $\mathrm{C}_{\max }-\mathrm{C}_{\min }$. The load was in the off-state $(100 \mathrm{G} \Omega$ ) between these capacitance values. The state was switched smoothly to avoid numerical instabilities.

The MEMS work-function energy harvester with capacitive comb electrodes ${ }^{31}$ was simulated using a Simulink ${ }^{\circledR}$ model consisting of the coupled equations (2), (5), (7), (8), and $V_{g a p}=u-V_{b i}$. The capacitance of the MEMS device was calculated with equation (5), where $\mathrm{d}(\mathrm{t})=\mathrm{x}(\mathrm{t})+\mathrm{x}_{0}$, and $\mathrm{x}_{0}$ is the rest position of the system. The spatial gradient of $\mathrm{C}_{\text {gap }}$ needed in the calculation of the electrostatic force was calculated with $\nabla C_{g a p}=-\varepsilon_{0} A / d(t)$. The following parameter values were used in the MEMS simulations ${ }^{31}$ : proof mass $\mathrm{m}=1.76 \cdot 10^{-5} \mathrm{~kg}$, spring constant $\mathrm{k}=2425 \mathrm{~N} / \mathrm{m}$, electrode area $\mathrm{A}=9 \cdot 10^{-5} \mathrm{~m}^{2}$, and air gap at rest $\mathrm{x}_{0}=26 \mu \mathrm{m}$. An intrinsic damping coefficient $b$ of the simulated device was calculated using the formula for the quality factor $Q_{r}=2 \pi f_{r} m / b \approx 100$ and a value of $1 \mathrm{pF}$ was assumed for the parasitic capacitance.

The case of the variable load was simulated by adding an electric switch into our Simulink ${ }^{\circledR}$ model. Switching of the load resistance between 50 G $\Omega$ (off-state) to $100 \Omega$ (on-state) was controlled by the time derivative of the capacitance. For better stability the time derivative was used to control the switch instead of the capacitance itself. Charging of the work capacitor took place when $\mathrm{dC} / \mathrm{dt}<0.01 \cdot \max (\mathrm{dC} / \mathrm{dt})$ and discharging when $\mathrm{dC} / \mathrm{dt}>-0.002 \cdot \max (\mathrm{dC} / \mathrm{dt})$.

1. White Jr, B. E. Energy-harvesting devices: Beyond the battery. Nat. Nanotechnol. 3, 71-72 (2008).

2. Puccinelli, D. \& Haenggi, M. Wireless Sensor Networks: Applications and Challenges of Ubiquitous Sensing. IEEE Circuits Syst. Mag. 5, 19 (2005).

3. Tobón, D. P., Falk, T. H. \& Maier, M. Context awareness in WBANs: a survey on medical and non-medical applications. IEEE Wireless Commun. 20, 30 (2013).

4. Joshi, G. P., Nam, S. Y. \& Kim, S. W. Cognitive Radio Wireless Sensor Networks: Applications, Challenges and Research Trends. Sensors 13, 11196 (2013).

5. Malik, B. \& Singh, V. R. A survey of research in WBAN for biomedical and scientific applications. Health Technol. 3, 227 (2013).
6. Beeby, S. P., Tudor, M. J. \& White, N. M. Energy harvesting vibration sources for microsystems applications. Meas. Sci. Technol. 17, R175 (2006).

7. Dario, P., Carrozza, M. C., Benvenuto, A. \& Menciassi, A. Microsystems in biomedical applications. J. Micromech. Microeng. 10, 235 (2000).

8. Pang, C., Lee, C. \& Suh, K.-Y. Recent advances in flexible sensors for wearable and implantable devices. J. Appl. Polym. Sci. 130, 1429 (2013).

9. Pfenniger, A., Jonsson, M., Zurbuchen, A., Koch, V. M. \& Vogel, R. Energy Harvesting from the Cardiovascular System, or How to Get a Little Help from Yourself. Ann. Bio. Eng. 41, 2248 (2013).

10. Cook-Chennault, K. A., Thambi, N. \& Sastry, A. M. Powering MEMS portable devices-a review of non-regenerative and regenerative power supply systems with special emphasis on piezoelectric energy harvesting systems. Smart Mater. Struct. 17, 043001 (2008).

11. Roundy, S. On the Effectiveness of Vibration Based Energy Harvesting. J. Int. Mat. Syst. Str. 16, 809 (2005).

12. Cottone, F., Vocca, H. \& Gammaitoni, L. Nonlinear Energy Harvesting. Phys. Rev. Lett. 102, 080601 (2009).

13. Dragunov, V. P. \& Ostertak, D. I. Microelectromechanical Converters. Russ. Microelectron. 41, 120 (2011).

14. Wang, L. \& Yuan, F. G. Vibration energy harvesting by magnetostrictive material. Smart Mater. Struct. 17, 045009 (2008).

15. Karaman, I., Basaran, B., Karaca, H. E., Karsilayan, A. I. \& Chumlyakov, Y. I. Energy harvesting using martensite variant reorientation mechanism in a NiMnGa magnetic shape memory alloy. Appl. Phys. Lett. 90, 172505 (2007).

16. Zhu, G., Chen, J., Zhang, T., Jing, Q. \& Wang, Z. L. Radial-arrayed rotary electrification for high performance triboelectric generator. Nat. Commun. 5 , 3426 (2014).

17. Suzuki, Y., Miki, D., Edamoto, M. \& Honzumi, M. A MEMS electret generator with electrostatic levitation for vibration-driven energy-harvesting applications. J. Micromech. Microeng. 20, 104002 (2010).

18. Lord Kelvin, V. Contact electricity of metals. Philos. Mag. Series 5 Vol. 46, 82-120 (1898).

19. Thomson, W. On a Method of Measuring Contact Electricity. Nature 23, 567-568 (1881).

20. Sze, S. M. Physics of Semiconductor Devices [2 ${ }^{\text {nd }}$ ed.] (Wiley-Interscience, New York, 1981).

21. Fonash, S. J. Solar Cell Device Physics [2 $2^{\text {nd }}$ ed.] (Academic Press, Oxford, 2010).

22. Schwede, J. W. et al. Photon-enhanced thermionic emission for solar concentrator systems. Nat. Mater. 9, 762-767 (2010).

23. Varpula, A. \& Prunnila, M. Diffusion-emission theory of photon enhanced thermionic emission solar energy harvesters. J. Appl. Phys. 112, 044506 (2012).

24. Kuehne, I., Frey, A., Marinkovic, D., Eckstein, G. \& Seidel, H. Power MEMS-A capacitive vibration-to-electrical energy converter with built-in voltage. Sensor. Actuat. A-Phys. 142, 263 (2008).

25. Li, L., Zhang, Y., San, H., Guo, Y. \& Chen, X. MEMS for vibration energy harvesting. Proc. SPIE 6836, 683610 (2008).

26. Dragunov, V. P. \& Lyutaeva, M. N. Parameters Estimation of the MEM Transducer with Electrodes Produced from Different Materials. Int. School and Seminar Internano'2009, pp. 93-96 (IEEE, Novosibirsk, 2009).

27. Lite, D. R. (ed.-in-chief). CRC Handbook of chemistry and physics [89 $9^{\text {th }}$ ed.] (CRC, Boca Raton, 2008).

28. Kempitiya, A., Borca-Tasciuc, D.-A. \& Hella, M. M. Low-Power Interface IC for Triplate Electrostatic Energy Converters. IEEE T. Power Electr. 28, 609 (2013).

29. Torres, E. O. \& Rincón-Mora, G. A. A 0.7- $\mu \mathrm{m}$ BiCMOS Electrostatic EnergyHarvesting System IC. IEEE J. Solid-St. Circ. 45, 483 (2010).

30. Torres, E. O. \& Rincón-Mora, G. A. Self-Tuning Electrostatic Energy-Harvester IC. IEEE T. Circuits-II: Exp. Briefs 57, 808 (2010).

31. Chiu, Y. \& Tseng, V. F. G. A capacitive vibration-to-electricity energy converter with integrated mechanical switches. J. Micromech. Microeng. 18, 104004 (2008)

32. Gorelik, L. Y. et al. Shuttle Mechanism for Charge Transfer in Coulomb Blockade Nanostructures. Phys. Rev. Lett. 80, 4526 (1998).

33. Roundy, S., Wright, P. K. \& Pister, K. S. J. Micro-electrostatic vibration-toelectricity converters. ASME International Mechanical Engineering Congress and Exposition, IMECE2002, Proceedings, 487-496 (2002).

34. Tang, L., Yang, Y. \& Kiong Soh, C. [Broadband Vibration Energy Harvesting Techniques] Advances in Energy Harvesting Methods [Elvin, N., Erturk A. (eds.)][17-61] (Springer, New York, 2013).

35. He, T. et al. Silicon Carbide (SiC) Nanoelectromechanical Switches and Logic Gates with Long Cycles and Robust Performance in Ambient Air and at High Temperature. 2013 IEEE International Electron Devices Meeting (Washington D.C., 9-11 Dec. 2013).

36. Simon, P. \& Gogotsi, Y. Materials for electrochemical capacitors. Nat. Mater. 7, 845-854 (2008)

37. Kaajakari, V. Practical MEMS (Small Gear Publishing, Las Vegas, 2009).

\section{Acknowledgments}

Fruitful discussions with Ingmar Stuns, Arto Rantala, Andrey Shchepetov, and James R. Dekker are gratefully acknowledged. 


\section{Author contributions}

A.V. and M.P. developed the theoretical models. A.V., T.H., and M.P. designed and built the macroscopic prototype of the WFEH. T.H. fabricated the samples. S.J.L. set up the data acquisition system. S.J.L. and A.V. designed and performed the experiments and analysed the data. J.K. performed the MEMS simulations. A.V. wrote the manuscript. M.P. supervised the project and edited the manuscript. All authors discussed the results and implications and commented on the manuscript at all stages.

\section{Additional information}

Supplementary information accompanies this paper at http://www.nature.com/ scientificreports

Competing financial interests: The authors declare no competing financial interests.
How to cite this article: Varpula, A., Laakso, S.J., Havia, T., Kyynäräinen, J. \& Prunnila, M. Harvesting Vibrational Energy Using Material Work Functions. Sci. Rep. 4, 6799; DOI:10.1038/srep06799 (2014).

(c) (i) (2) (2) This work is licensed under a Creative Commons Attribution-NonCommercialShareAlike 4.0 International License. The images or other third party material in this article are included in the article's Creative Commons license, unless indicated otherwise in the credit line; if the material is not included under the Creative Commons license, users will need to obtain permission from the license holder in order to reproduce the material. To view a copy of this license, visit http:// creativecommons.org/licenses/by-nc-sa/4.0/ 